\title{
Analytical time-domain model for radio over free space optical (RoFSO) systems considering scintillation effect
}

\author{
Wansu Lim, Student Member, IEEE, Tae-Sik Cho, Changho Yun, \\ Pandelis Kourtessis Member, IEEE and Kiseon Kim, Senior Member, IEEE
}

\begin{abstract}
In this paper, an analytical time-domain model is presented to analyze the radio over free space optical (RoFSO) systems considering scintillation effect with the log-normal distribution. This analytical model uses general cases of a dual-drive Mach-Zehnder modulator (DD-MZM) and photodetector (PD) for typical optical double sideband (ODSB) and single sideband (OSSB) signals. In addition, we show output current of PD as a function of the summation of each frequency component in time domain. Finally, we calculate the received signal power with the power spectral density (PSD) and derive a closed-form average BER performance.
\end{abstract}

\section{Index Terms}

Free space optical (FSO) systems, radio over free space optical (RoFSO) systems, dual drive MachZehnder modulator, optical single sideband (OSSB), optical double sideband (ODSB), closed form, time domain.

\section{INTRODUCTION}

Recently, free space optical (FSO) systems are actively researched to support the future broadband access network [1]-[4]. Especially, FSO systems is a necessary technology for tactical

Wansu Lim and Pandelis Kourtessis are with the Department of Engineering and Technology, University of Hertfordshire, UK, (e-mail: w.lim9@herts.ac.uk)

Tae-Sik Cho and Kiseon Kim are with the Department of Information and Communications, Gwangju Institute of Science and Technology, South Korea

Changho Yun is with Korea Ocean Research and Development Institute (KORDI), South Korea 
military communications due to the increasing bandwidth requirements particularly for the transmission of high resolution imagery acquired by tactical sensors [5]. The United States Department of Defense Advanced Research Projects Agency (DARPA) Strategic Technology Office has specifically proposed the Optical RF Communications Adjunct (ORCA) [6]. Subsequently, there are reported many works on radio over FSO (RoFSO) systems, useful and necessary in the military communications [7]-[9].

In RoFSO systems, a dual-drive Mach-Zehnder modulator (DD-MZM) has been widely used for high data rate services since it is robust against laser chirp and provides high spectral efficiency [10]. Moreover, for analyzing complex nonlinear RoFSO systems with DD-MZM, analytical model is used for a simplified estimation and optimization of a system performance [11], [12]. For modeling the nonlinear part consisted of a DD-MZM and photodetector (PD), there are two approaches which are based on frequency domain and time domain.

In a frequency domain based approach, the calculation of the convolution between input signal in time domain and channel transfer function can be easily obtained by multiplication in frequency domain. Additionally, it is easy to handle pure carrier signals since it can be simply expressed as delta functions. [12] shows an exact analytical model with a closed form for fiber optic links with general parameters of a DD-MZM in frequency domain, which solved complex equations simply and efficiently by utilizing Fourier transform of an optical field signal at the end of optical fiber and Graf's addition theorem. Subsequently, the output photocurrent including harmonics can be clearly expressed in [12].

In a time domain based approach, it is often difficult to numerically evaluate the convolution since it requires infinite sum or integration. Furthermore, a time domain approach can generate infinite sum of signals [11]. In spite of the above challenges, time domain based approach has an alternative advantage to deal with complicated input signals which have been difficult to express and handle in frequency domain such as phase noises from a laser and RF oscillator [13], [14]. Thus, [13] and [14] straightforwardly analyzed the system performance in time domain, and at the last step of analysis, they utilized the power spectrum density to investigate the characteristics of a received signal. Therefore, the time domain approach to model the entire RoFSO systems can be used for efficiently analyzing the system as an alternative if the output of a channel can be easily evaluated and final results can be expressed with a closed form.

In this paper, we present a time domain model for nonlinear optical link incorporating general 
parameters of a DD-MZM such as dc bias, phase shift, and each amplitude to each arm of a DD-MZM in conjunction with a photodetector. Also, we derive a closed-form average BER performance considering scintillation effect using the log-normal distribution. For the application of this analytical model, we show simple typical modulation cases: optical single sideband (OSSB) and optical double sideband (ODSB). Additionally, the power spectral density (PSD) of the photocurrent is shown to describe the characteristics of a photocurrent in detail.

\section{Channel MODEL}

FSO systems basically utilize free atmospheric space medium for transmission, and are inherently affected by atmospheric conditions. Especially, in this paper we consider the scintillation effect influencing the signal fading. Subsequently, its an interesting problem to analyze the degraded signal strength and link performance against scintillation effect.

We use the log-normal distribution which well represents scintillation. The log-normal distribution is modeled as follows [15], [16]:

$$
f_{\varphi}(\varphi)=\frac{1}{2 \varphi \sqrt{2 \pi \sigma_{k}^{2}}} \exp \left(-\frac{\left(\ln (\varphi)+2 \sigma_{k}^{2}\right)^{2}}{8 \sigma_{k}^{2}}\right), \varphi>0
$$

where $\varphi$ is the scintillation coefficient and $\sigma_{k}$ is the standard deviation of $K, K=\ln (\varphi) / 2$. According to the atmospheric conditions, $\sigma_{k}^{2}$ is defined as

$$
\sigma_{k}^{2}=\exp \left[\frac{0.49 \sigma_{R}^{2}}{\left(1+0.18 d^{2}+0.56 \sigma_{R}^{12 / 5}\right)^{7 / 6}}+\frac{0.51 \sigma_{R}^{2}}{\left(1+0.9 d^{2}+0.62 d^{2} \sigma_{R}^{12 / 5}\right)^{5 / 6}}\right]-1
$$

where $d=\left(\kappa D^{2} / 4 L\right)^{1 / 2}, D$ is the diameter of the receiver collecting lens aperture, $L$ is the link distance in meters, $\kappa=2 \pi / \lambda$ is the optical wave number, $\lambda$ is the wavelength and $\sigma_{R}^{2}$ is Rytov variance which has been used as an estimate of the intensity variance. $\sigma_{R}^{2}$ is given by

$$
\sigma_{R}^{2}=1.23 C_{n}^{2} \kappa^{7 / 6} L^{11 / 6}
$$

$C_{n}^{2}$ is the strength of the atmospheric turbulence and stands for the altitude-dependent index of the refractive structure parameter. The scintillation index $(S I)$ is defined as $S I=e^{4 \sigma_{k}^{2}}-1$.

\section{ANALYTICAL TIME-DOMAIN MODEL FOR THE RADIO OVER FSO SYSTEMS}

Fig. 1 shows the overall architecture of the RoFSO system. A RF signal from the RF modulator is split by a phase shifter. This RF signal is injected directly into the DD-MZM as shown in (6). 


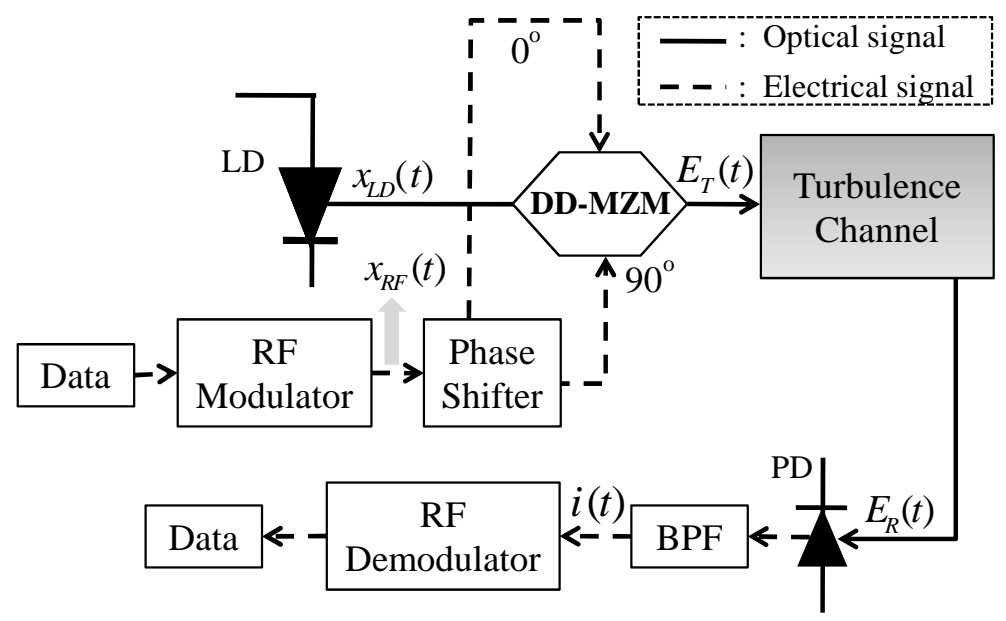

Fig. 1. Overall architecture of the radio over FSO system considering of optical transmitter, turbulence channels, and optical receiver.

The output signal of the DD-MZM is transmitted via atmospheric turbulence channels between telescopes. The received signals are detected by the photodetector (PD), and the photocurrent corresponding to the transmitted RF signal is extracted by the bandpass filter (BPF). Finally, data is extracted by the RF demodulator module. The optical signal, $x_{L D}(t)$ from the laser and the RF signal, $x_{R F}(t)$ from the RF modulator are modeled respectively, as follows:

$$
\begin{aligned}
& x_{L D}(t)=A_{0} \cdot \exp \left(j \omega_{L D} t\right) \\
& x_{R F}(t)=V_{R F} \cdot \cos \left(\omega_{R F} t\right)
\end{aligned}
$$

where $A_{0}$ and $V_{R F}$ define amplitudes from a laser diode (LD) and RF oscillator, $\omega_{L D}$ and $\omega_{R F}$ are angular frequencies of the signals. After optically modulating $x_{L D}(t)$ by $x_{R F}(t)$ with a DD-MZM, the output signal of a DD-MZM is represented as [14]

$$
\begin{aligned}
E_{T}(t) & =\frac{L_{a t t} \cdot x_{L D}(t)}{2} \times\left\{\exp \left[j \frac{\pi \gamma \cdot \tilde{x}_{R F}(t)}{V_{\pi}}+j \theta\right]+\exp \left[j \frac{\pi \sqrt{1-\gamma^{2}} \cdot x_{R F}(t)}{V_{\pi}}\right]\right\} \\
& =A_{0} \cdot e^{j \omega_{L D} t} \sum_{n=-\infty}^{\infty} a_{n} \cdot \exp \left(j n \omega_{R F} t\right)
\end{aligned}
$$

where $a_{n}=j^{n}\left[J_{n}\left(\beta_{1}\right) \exp \left(j n \theta_{1}+j \theta\right)+J_{n}\left(\beta_{2}\right)\right] . \theta\left(=V_{d c} / V_{\pi}\right), V_{d c}$ and $V_{\pi}$ are a normalized DC, a bias voltage and a switching voltage of a DD-MZM, respectively. $L_{a t t}\left(=10^{-L_{D M} / 20}\right)$ is the attenuation of DD-MZM due to an insertion loss $L_{D M} . \tilde{x}_{R F}(t)$ is the phase-shifted version 
of $x_{R F}(t) . \beta_{1}\left(=\pi \gamma V_{R F} / V_{\pi}\right)$ and $\beta_{2}\left(=\pi \sqrt{1-\gamma^{2}} V_{R F} / V_{\pi}\right)$ are the modulation indexes for each arm and $\gamma$ is the ratio of a power splitter.

After the transmission of turbulence channels, the received optical signal is expressed as

$$
E_{R}(t)=\sqrt{\varphi} \cdot A_{0} \cdot e^{j \omega_{L D} t} \sum_{n=-\infty}^{\infty} a_{n} \exp \left(j n \omega_{R F} t\right) .
$$

By using a square law model, the photocurrent $i(t)$ can be obtained as follows:

$$
\begin{aligned}
i(t)= & \Re\left|E_{R}(t)\right|^{2}+n(t) \\
= & \Re \varphi A_{0}^{2} \sum_{n=-\infty}^{\infty} \sum_{m=-\infty}^{\infty} j^{n+3 m} e^{j\left[(n-m) \omega_{R F} t\right]}\left\{J_{n}\left(\beta_{1}\right) J_{m}\left(\beta_{1}\right) e^{j(n-m) \theta_{1}}+J_{n}\left(\beta_{1}\right) J_{m}\left(\beta_{2}\right) e^{j\left(n \theta_{1}+\theta\right)}\right. \\
& \left.+J_{n}\left(\beta_{2}\right) J_{m}\left(\beta_{1}\right) e^{-j\left(m \theta_{1}+\theta\right)}+J_{n}\left(\beta_{2}\right) J_{m}\left(\beta_{2}\right)\right\}+n(t)
\end{aligned}
$$

where $\Re$ is the responsivity of a photodetector (PD) and $n(t)$ are additive noises such as thermal and shot noises. For simplifying (7), the Graf's addition theorem [17] and $n=m+a$ are utilized. It is noteworthy that substituting $m+a$ for $n$ is necessary to make double infinite sum independent and can be extendedly applied in multicarrier to reduce an infinite sum. By using the method, (7) is reduced to

$$
\begin{gathered}
i(t)=\Re \varphi A_{0}^{2} \sum_{a=-\infty}^{\infty} g_{a} \cdot e^{j a \omega_{R F} t}+n(t) \\
g_{a}=j^{a}\left[e^{-j a\left(-\theta_{1}+\Psi_{1}\right)} J_{a}\left(Z_{1}\right)+e^{-j a\left(-\theta_{1}+\Psi_{2}\right)} e^{j \theta} J_{a}\left(Z_{2}\right)+e^{-j a\left(\Psi_{3}\right)} e^{-j \theta} J_{a}\left(Z_{3}\right)+e^{-j a\left(\Psi_{4}\right)} J_{a}\left(Z_{4}\right)\right] \\
Z_{i}=\sqrt{u_{i}^{2}+v_{i}^{2}-2 u_{i} v_{i} \cos \left(\alpha_{i}\right)} \\
u_{i}-v_{i} \cos \left(\alpha_{i}\right)=Z_{i} \cos \left(\Psi_{i}\right) \\
v_{i} \sin \left(\alpha_{i}\right)=Z_{i} \sin \left(\Psi_{i}\right)
\end{gathered}
$$

where $u_{1}=v_{1}=\beta_{1}$ and $\alpha_{1}=0$ for $Z_{1}, u_{2}=\beta_{1}, v_{2}=\beta_{2}$, and $\alpha_{2}=-\theta_{1}$ for $Z_{2}, u_{3}=\beta_{2}$, $v_{3}=\beta_{1}$, and $\alpha_{3}=\theta_{1}$ for $Z_{3}, u_{4}=\beta_{2}, v_{4}=\beta_{2}$, and $\alpha_{4}=0$ for $Z_{4}$, and $f_{R F}\left(=\omega_{R F} / 2 \pi\right)$ is the frequency of the input RF signal. It is noticeable that the form of the output photocurrent $i(t)$ in (8) is similar to the form of the input $E_{T}(t)$. 


\section{A. Optical double sideband modulation (ODSB)}

For ODSB modulation, $\beta_{1}=\beta_{2}=\beta, \theta_{1}=\pi$, and $\Psi_{1}=\Psi_{2}=\Psi_{3}=\Psi_{4}=0$. From (8), we can obtain the photocurrent, $i_{d}(t)$, for ODSB signals as follows:

$$
i_{d}(t)=\Re \varphi A_{0}^{2} \sum_{a=-\infty}^{\infty} d_{a} \cdot e^{j a \omega_{R F} t}+n(t)
$$

where $d_{a}=j^{a}\left[(-1)^{a}+1\right] J_{a}(0)+j^{a}\left[j(-1)^{a}-1\right] J_{a}(2 \beta)$. With (13), we can straightforwardly obtain the PSD of a photocurrent in ODSB case as follows:

$$
\begin{aligned}
P_{d} & =F\left\{\left\langle i_{d}^{*}(t) \times i_{d}(t+\tau)\right\rangle\right\} \\
& =\Re^{2} \varphi^{2} A_{0}^{4} \sum_{a=-\infty}^{\infty}\left|d_{a}\right|^{2} \cdot \delta\left(f-a f_{R F}\right)
\end{aligned}
$$

where $*$ denotes complex conjugate, $\langle\cdot\rangle$ and $F\{\cdot\}$ denote mean and Fourier transfer functions, respectively.

\section{B. Optical single sideband modulation (OSSB)}

For OSSB modulation, $\beta_{1}=\beta_{2}=\beta, \theta_{1}=\pi / 2, \Psi_{1}=\Psi_{4}=0, \Psi_{2}=-\pi / 4$, and $\Psi_{3}=\pi / 4$. From (8), we can obtain the photocurrent, $i_{s}(t)$, for OSSB signals as follows:

$$
i_{s}(t)=\Re \varphi A_{0}^{2} \sum_{a=-\infty}^{\infty} s_{a} \cdot e^{j a \omega_{R F} t}+n(t),
$$

where $s_{a}=j^{a}\left[e^{j a \pi / 2}+1\right] J_{a}(0)+j^{a+1}\left[e^{j a 3 \pi / 4}-e^{-j a \pi / 4}\right] J_{a}(\sqrt{2} \beta)$. From (15), we can evaluate the PSD of a photocurrent in OSSB case as follows:

$$
P_{s}=\Re^{2} \varphi^{2} A_{0}^{4} \sum_{a=-\infty}^{\infty}\left|s_{a}\right|^{2} \cdot \delta\left(f-a f_{R F}\right) .
$$

\section{DERIVATION OF BER PERFORMANCE}

In this section, we derive a closed form of the average BER considering considering scintillation effect. First, we define the signal to noise ratio (SNR) as follows:

$$
S N R=\frac{P_{d}\left(\text { or } P_{s}\right)}{P_{t h}+P_{\text {shot }}} \simeq \frac{\Re^{2} \varphi^{2} A_{0}^{4} \Upsilon}{4 k T B+q \Re A_{0}^{2} \varphi B}
$$

where $\Upsilon=\sum_{a=-\infty}^{\infty}\left|d_{a}\right|^{2} \cdot \delta\left(f-a f_{R F}\right)$ for ODSB or $\sum_{a=-\infty}^{\infty}\left|s_{a}\right|^{2} \cdot \delta\left(f-a f_{R F}\right)$ for OSSB, $P_{t h}$ is the thermal noise power, $P_{\text {shot }}$ is the shot noise power, $k=1.38 \times 10^{-23} \mathrm{~J} / \mathrm{K}$ is the 
Boltzmann constant, $q=1.6 \times 10^{-19} C$ is the electron charge, $T$ is the absolute temperature, and $B$ is the effective noise bandwidth. Using the above SNR and scintillation effect, we then derive the average $\operatorname{BER}\left(P_{b}\right)$ as

$$
\begin{aligned}
P_{b} & =\int_{0}^{\infty} Q(\sqrt{S N R}) f_{\varphi}(\varphi) d_{\varphi} \\
& =\int_{0}^{\infty} Q\left(\sqrt{\frac{\Re^{2} \varphi^{2} A_{0}^{4} \Upsilon}{4 k T B+q \Re A_{0}^{2} \varphi B}}\right) \frac{1}{2 \varphi \sqrt{2 \pi \sigma_{k}^{2}}} \exp \left(-\frac{\left(\ln (\varphi)+2 \sigma_{k}^{2}\right)^{2}}{8 \sigma_{k}^{2}}\right) d_{\varphi} .
\end{aligned}
$$

Using the change of variable $x=\left(\ln \varphi+2 \sigma_{k}^{2}\right) / \sqrt{8} \sigma_{k}$ and the Gauss-Hermite quadrature formula [18], (18) can then be simplified as

$$
\begin{aligned}
P_{b} & =\frac{1}{2 \varphi \sqrt{2 \pi \sigma_{k}^{2}}} \int_{0}^{\infty} Q\left(\sqrt{\frac{\Re^{2} A_{0}^{4} \Upsilon e^{2\left(\sqrt{8} \sigma_{k} x_{i}-2 \sigma_{k}^{2}\right)}}{4 k T B+q \Re A_{0}^{2} B e^{\left(\sqrt{8} \sigma_{k} x_{i}-2 \sigma_{k}^{2}\right)}}}\right) \exp \left(-x^{2}\right) d_{x} \\
& =\frac{1}{\sqrt{\pi}} \sum_{i=1}^{N} w_{i} Q\left(\sqrt{\frac{\Re^{2} A_{0}^{4} \Upsilon e^{2\left(\sqrt{8} \sigma_{k} x_{i}-2 \sigma_{k}^{2}\right)}}{4 k T B+q \Re A_{0}^{2} B e^{\left(\sqrt{8} \sigma_{k} x_{i}-2 \sigma_{k}^{2}\right)}}}\right)
\end{aligned}
$$

where $N$ is the order of approximation, $x_{i}, i=1, \ldots, N$ are the zeros of the $N$ th-order Hermite polynormial and $w_{i}, i=1, \ldots, N$ are weight factors for the $N$ th-order approximation; $N=10$ is used for the analysis [19].

\section{NumericAl Results}

In this section, we present numerical results for the BER performance of RoFSO systems according to various metrics such as the scintillation index $(S I)$, propagation distance $(L), C_{n}^{2}$, temperature $(T)$, the power of the laser diode, and wavelength $(\lambda)$. The system parameters used in the analysis are described in Table I.

Fig. 2 illustrates the results of the average BER as a function of the scintillation index under the log-normal channel for ODSB according to three different temperature (e.g, $240 \mathrm{~K}, 290$ $\mathrm{K}, 340 \mathrm{~K})$. Generally, the transceivers of FSO systems are installed on rooftops so that they 
TABLE I

ROFSO SYSTEM PARAMETERS.

\begin{tabular}{|c||c|}
\hline \multicolumn{1}{|c||}{ Parameter } & Value \\
\hline \hline Wavelength of LD $(\lambda)$ & $850 \mathrm{~nm}$ or $1550 \mathrm{~nm}$ \\
\hline Diameter of receiver & $3 \mathrm{~cm}$ \\
\hline Date rate & $1.2 \mathrm{Gbps}$ \\
\hline Switching voltage $\left(V_{\pi}\right)$ & $2.5 \mathrm{~V}$ \\
\hline DD-MZM insertion loss $\left(L_{D M}\right)$ & $6 \mathrm{~dB}$ \\
\hline Ratio of a power splitter $(\gamma)$ & $\sqrt{0.5}$ \\
\hline Normalized DC $(\theta)$ & $\pi / 2$ \\
\hline Responsivity $(\Re)$ & $0.6 \mathrm{~A} / \mathrm{W}$ \\
\hline
\end{tabular}

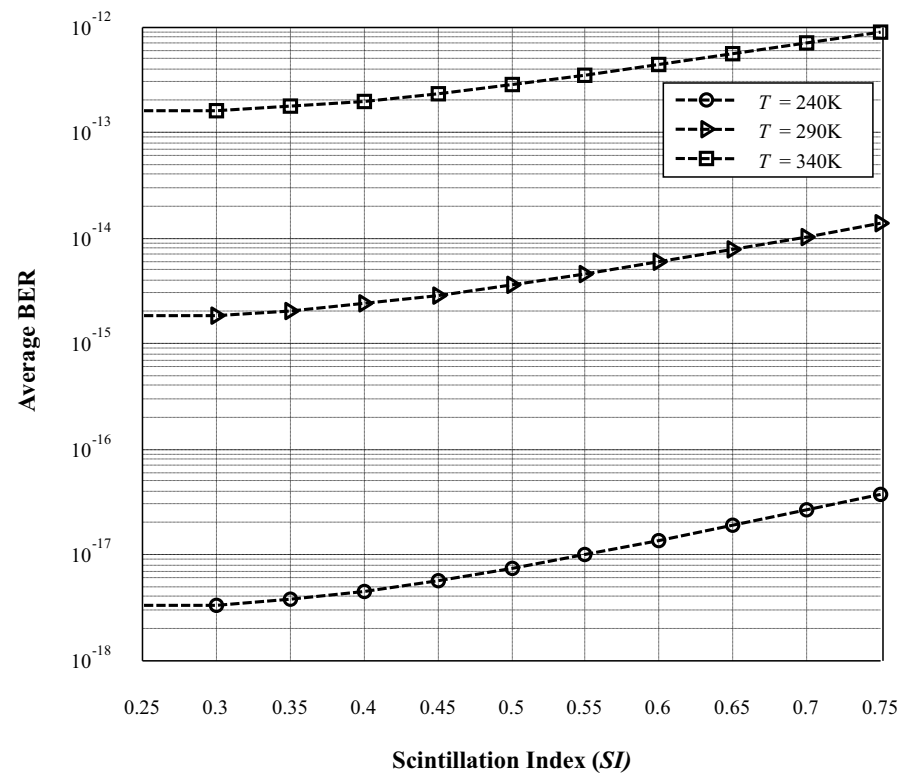

Fig. 2. Average BER as a function of the scintillation index under the log-normal channel for ODSB with three different temperatures (e.g., $240 \mathrm{~K}, 290 \mathrm{~K}, 340 \mathrm{~K}$ ).

are affected by the temperature of environment. As shown in Fig. 2, with the increase of the temperature the BER performance for $S I$ between 0.25 and 0.75 is getting worse.

Fig. 3 represents the relationship the average BER and the propagation distance for ODSB according to two different wavelengthes $(850 \mathrm{~nm}$ and $1550 \mathrm{~nm})$. Using the relationship between Rytov variance $\left(\sigma_{R}^{2}\right)$ and the optical wave number $(\kappa=2 \pi / \lambda)$ as shown in (3), we can expect 


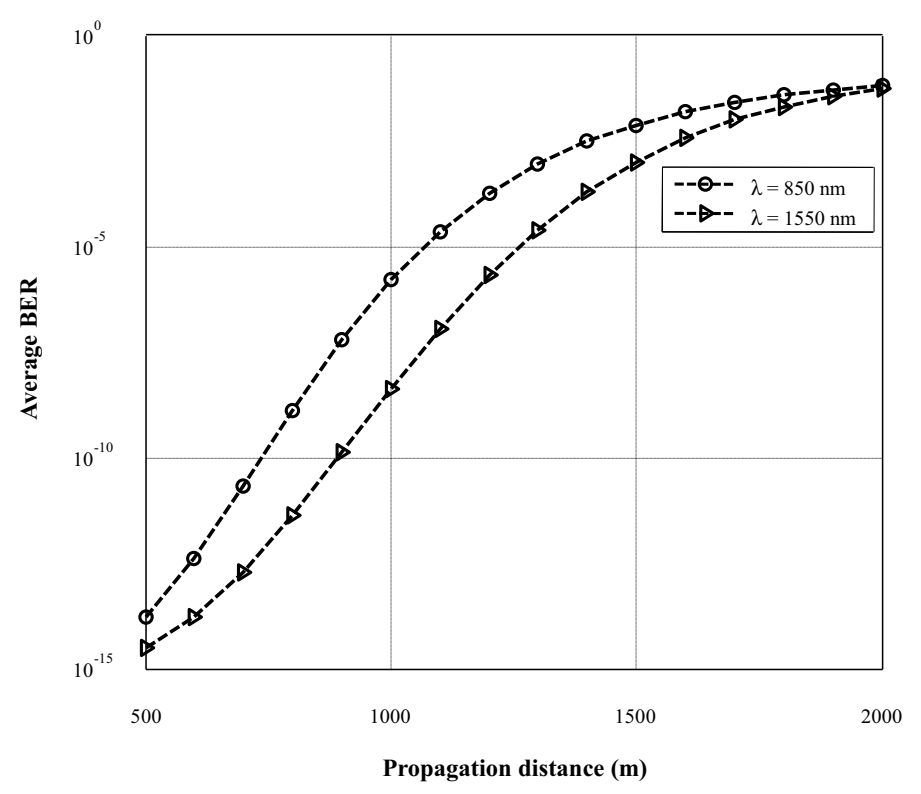

Fig. 3. Average BER as a function of the propagation distance for ODSB according to two different wavelengths (e.g., 850 $\mathrm{nm}, 1550 \mathrm{~nm})$.

the influence of the wavelength to the FSO systems. As a result, we confirm that wavelength affects the system performance and the systems using $1550 \mathrm{~nm}$ has the better performances than that using $850 \mathrm{~nm}$.

Since the turbulence channel is sensitive to $C_{n}^{2}$ as shown in (3), we represent the result of the average BER as a function of $C_{n}^{2}$ according to three different propagation distances (e.g, 1000 m, $1500 \mathrm{~m}, 2000 \mathrm{~m}$ ) for OSSB in Fig. 4. Furthermore, as the propagation distance increases, the average BER increases rapidly since the long distance makes the turbulence channel strong. Also, it is shown that the difference of average BER corresponding to $L=1.5 \mathrm{Km}$ and $L=1$ $\mathrm{Km}$ is almost 3.1 times larger than that between $L=2 \mathrm{Km}$ and $L=1.5 \mathrm{Km}$ at a given $C_{n}^{2}$ of $3 \times 10^{-14}$.

\section{CONCLUSiOnS}

In this paper, we propose an analytical time-domain model for the radio over FSO systems using a DD-MZM and PD. This model can be also extended to another link required to a time domain such as phase noise from a laser and RF oscillator since this analysis is directly evaluated in time domain. Additionally, this approach can be easily modified for multicarrier input 


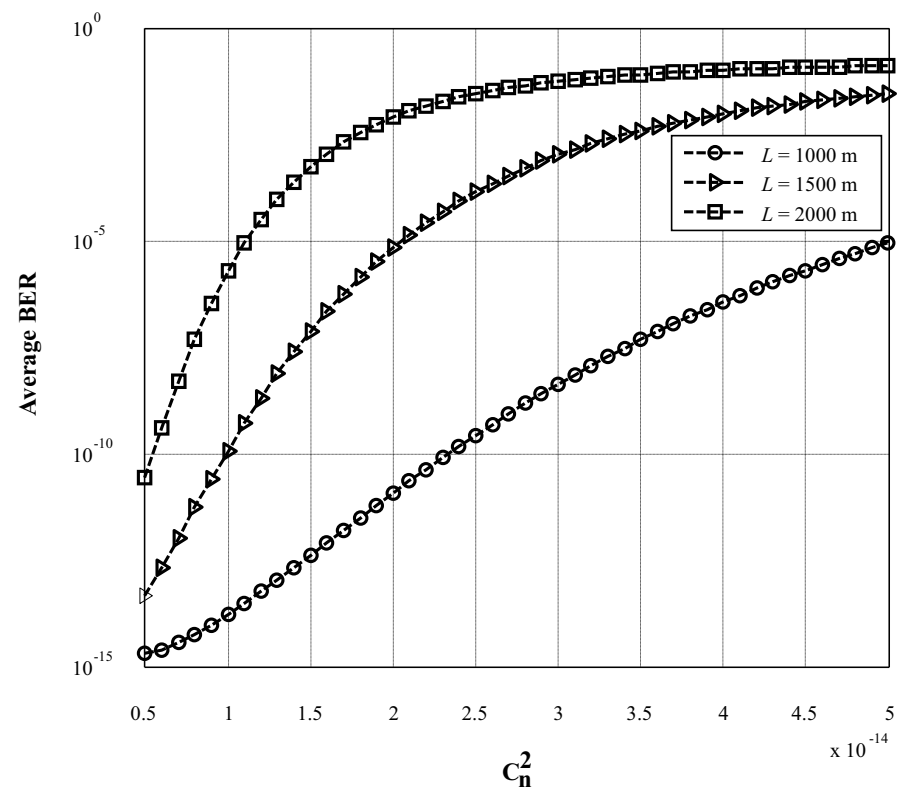

Fig. 4. Average BER as a function of $C_{n}^{2}$ for OSSB with three different propagation distances (e.g, $1000 \mathrm{~m}, 1500 \mathrm{~m}, 2000 \mathrm{~m}$ ).

signals because no complicated convolution is required. Also, we derive a closed-form average BER performance considering scintillation effect using the log-normal distribution based on the Gauss- Hermite quadrature formula for ODSB and OSSB. As a result, we could more easily predict BER performance without requiring complicated calculations. In practical terms, when establishing RoFSO systems, we could construct an engineering table using this derived BER formula according to various conditions.

\section{ACKNOWLEDGEMENTS}

This work was partially supported by the Center for Distributed Sensor Networks at the Gwangju Institute of Science and Technology (GIST), by the World Class University (WCU) program (R31-20008-000-10026-0), and by a grant (B01-03) from the Plant Technology Advancement Program funded by the Ministry of Construction and Transportation, Korea.

\section{REFERENCES}

[1] A. García-Zambrana, C. Castillo-Vázquez, and B. Castillo-Vázquez, "Space-time trellis coding with transmit laser selection for FSO links over strong atmospheric turbulence channels," Opt. Express, vol. 18, pp. 5356-5366, June 2010.

[2] I. B. Djordjevic and G.T. Djordjevic "On the communication over strong atmospheric turbulence channels by adaptive modulation and coding," Opt. Express, vol. 17, pp. 18250-18262, Sep. 2009. 
[3] W. O. Popoola and Z. Ghassemlooy, "BPSK subcarrier intensity modulated free-space optical communications in atmospheric turbulence," J. Lightwave Technol., vol. 27, pp. 967-973, Apr. 2009.

[4] W. Lim, T. Cho, C. Yun, and K. Kim, "Average BER analysis of SCM-based free-space optical systems by considering the effect of IM3 with OSSB signals under turbulence channels," Opt. Express, vol. 17, pp. 20721-20726, Oct. 2009.

[5] R. S. Raghavan, A. Kam, and R. Y. Mannepalli, "Modeling \& simulation to study the performance of hybrid free space opticalirf military communication networks," in Proc. Military Communications Conference 2008 (MILCOM 2008), pp. 1-7, Nov. 2008.

[6] http://www.darpa.mil/sto/programs/orca/index.html

[7] L. B. Stotts, L. C. Andrews, P. C. Cherry, J. J. Foshee, P. J. Kolodzy, W. K. McIntire, M. Northcott, R. L. Phillips, H. A. Pike, B. Stadler and D. W. Young, "Hybrid Optical RF Airborne Communications," Proc. of the IEEE, vol. 97, pp. 1109-1127, June 2009.

[8] K. Kazaura, K. Wakamori, M. Matsumoto, T. Higashino, K. Tsukamoto, and S. Komaki, "RoFSO: A Universal Platform for Convergence of Fiber and Free-Space Optical Communication Networks," IEEE Commun. Mag., vol. 48, pp. 130-137, Feb. 2010.

[9] W. Lim, C. Cho, C. Yun, and K. Kim, "BER performance analysis of radio over free-space optical systems considering laser phase noise under Gamma-Gamma turbulence channels,” Opt. Express, vol. 17, no. 6, pp. 4479-4484, Mar. 2009.

[10] E. L. Wooten, "A review of lithium niobate modulators for fiber-optic communications systems," IEEE Photon. Technol. Lett., vol. 6, no. 1, pp. 69-82, Jan.-Feb. 2000.

[11] J. L. Corral, J. Marti, and J. M. Fuster, "General expressions for IM/DD dispersive analog optical links with external modulation or optical up-conversion in Mach-Zehnder electrooptical modulator," IEEE Trans. Microw. Theory Tech., vol. 49, no. 10, pp. 1968-1976, 2001.

[12] L. Cheng, S. Aditya, and A. Nirmalathas, "An exact analytical model for dispersive transmission in microwave fiber-optic links using Mach-Zehnder external modulator,” IEEE Photon. Technol. Lett., vol. 17, no. 7, pp. 1525-1527, July 2005.

[13] P. B. Gallion and G. Debarge, "Quantum phase noise and field correlation in single frequency semiconductor laser systems," IEEE J. Quantum Electron., vol. QE-6, no. 4, pp. 343-349, Apr. 1984.

[14] T-S. Cho, C. Yun, J-I. Song, and K. Kim, "Analysis of CNR penalty of radio over fiber systems including the effects of phase noise from laser and RF oscillator ," J. Lightwave Technol., vol. 23, no. 12, pp. 4093-4100, Dec. 2005.

[15] H. E. Nistazakis, E.A. Karagianni, A.D. Tsigopoulos, M.E. Fafalios, and G.S. Tombras, "Average capacity of optical wireless communication systems over atmospheric turbulence channels," J. Lightwave Technol., vol. 27, no. 8, pp. 974-979, Apr. 2009.

[16] N. Cvijetic, G.Wilson, M. Brandt-Pearce, "Performance bounds for free-space optical MIMO systems with APD receivers in atmospheric turbulence," J. Lightwave Technol., vol. 26, no. 3, pp. 3-12, Apr. 2008.

[17] M. Abramowitz and I. A. Stegun, Handbook of Mathematical Functions With Formulas, Graphs, and Mathematical Tables, 10th ed: Wiley, 1972.

[18] M. K. Simon and M. S. Alouini, Digital Communication over Fading Channels. (Wiley, New York, 2000).

[19] K. Kiasaleh, "Performance of APD-based, PPM free-space optical communication systems in atmospheric turbulence," IEEE Trans. Commun., vol. 53, no. 9, pp. 1455-1461, Apr. 2005. 